\title{
Systems of generalized Sturm-Liouville and Langevin fractional differential equations
}

\author{
Thanadon Muensawat ${ }^{1}$, Sotiris K Ntouyas ${ }^{2,3}$ and Jessada Tariboon ${ }^{1,4^{*}}$ (D)
}

\author{
"Correspondence: \\ jessada.t@sci.kmutnb.ac.th \\ ${ }^{1}$ Nonlinear Dynamic Analysis \\ Research Center, Department of \\ Mathematics, Faculty of Applied \\ Science, King Mongkut's University \\ of Technology North Bangkok, \\ Bangkok, 10800, Thailand \\ ${ }^{4}$ Centre of Excellence in \\ Mathematics, CHE, Sri Ayutthaya \\ Rd., Bangkok, 10400, Thailand \\ Full list of author information is \\ available at the end of the article
}

\begin{abstract}
In this paper, we study anti-periodic boundary value problems for systems of generalized Sturm-Liouville and Langevin fractional differential equations. Existence and uniqueness results are proved via fixed point theorems. Examples illustrating the obtained results are also presented.
\end{abstract}

MSC: 34A08; 34A12; 34B15

Keywords: Sturm-Liouville problem; Langevin equation; fractional differential equation; Caputo fractional derivative; boundary value problem

\section{Introduction}

Fractional differential equations have attracted the attention of many researchers working in a variety of disciplines due to the development and applications of these equations in many fields such as engineering, mathematics, physics, chemistry, etc. For recent development of the topic, we refer the reader to a series of books and papers [1-11]. The study of boundary value problems of coupled systems of fractional order differential equations is also very important as such systems appear in a variety of problems of applied nature, especially in biosciences, for instance, see [12-22].

The Langevin equation (first formulated by Langevin in 1908) is found to be an effective tool to describe the evolution of physical phenomena in fluctuating environments [23]. For some new developments on the fractional Langevin equation, see, for example, [2432]. The Sturm-Liouville problem has many applications in different areas of science, for example, engineering and mathematics. The classical Sturm-Liouville problem for a linear differential equation of second order is a boundary-value problem as the following one:

$$
\left\{\begin{array}{l}
-\frac{d}{d t}\left[p(t) \frac{d x}{d t}\right]+v(t) x=\lambda r(t) x, \quad t \in[a, b] \\
a_{1} x(a)+a_{2} x^{\prime}(a)=0 \\
b_{1} x(b)+b_{2} x^{\prime}(b)=0 .
\end{array}\right.
$$

Recently in [33], the authors proposed an approach to the fractional version of the Sturm-Liouville problem. They investigated the eigenvalues and eigenfunctions associated with these operators and also their properties with the objective of applying this generalized Sturm-Liouville theory to fractional partial differential equations.

(c) The Author(s) 2017. This article is distributed under the terms of the Creative Commons Attribution 4.0 International License (http://creativecommons.org/licenses/by/4.0/), which permits unrestricted use, distribution, and reproduction in any medium, provided you give appropriate credit to the original author(s) and the source, provide a link to the Creative Commons license, and indicate if changes were made. 
This paper investigates the existence of solutions for the following system of fractional differential equations:

$$
\begin{cases}D^{\alpha_{2}}\left(\left[p(t) D^{\alpha_{1}}+r(t)\right] x(t)\right)=f(t, x(t), y(t)), & 0<t<T, \\ D^{\beta_{2}}\left(\left[q(t) D^{\beta_{1}}+s(t)\right] y(t)\right)=g(t, x(t), y(t)), & 0<t<T,\end{cases}
$$

subject to anti-periodic boundary conditions

$$
\begin{cases}x(0)=-x(T), & D^{\alpha_{1}} x(0)=-D^{\alpha_{1}} x(T) \\ y(0)=-y(T), & D^{\beta_{1}} y(0)=-D^{\beta_{1}} y(T)\end{cases}
$$

where $D^{\theta}$ is the Caputo fractional derivative of orders $\theta \in\left\{\alpha_{1}, \alpha_{2}, \beta_{1}, \beta_{2}\right\}$ with $0<$ $\alpha_{1}, \alpha_{2}, \beta_{1}, \beta_{2}<1, f, g \in C\left([0, T] \times \mathbb{R}^{2}, \mathbb{R}\right), p, q \in C([0, T], \mathbb{R} \backslash\{0\})$ with $|p(t)| \geq K_{1},|q(t)| \geq$ $K_{2}, K_{1}, K_{2}>0$ and $r, s \in C([0, T], \mathbb{R})$.

Note that system (1.2) is a generalization of Sturm-Liouville and Langevin fractional differential systems. If $r(t), s(t) \equiv 0$ for all $t \in[0, T]$, then (1.2) is reduced to

$$
\begin{cases}D^{\alpha_{2}}\left(p(t) D^{\alpha_{1}} x(t)\right)=f(t, x(t), y(t)), & 0<t<T, \\ D^{\beta_{2}}\left(q(t) D^{\beta_{1}} y(t)\right)=g(t, x(t), y(t)), & 0<t<T,\end{cases}
$$

which are Sturm-Liouville fractional differential equations. If $p(t)=q(t) \equiv 1$ and $r(t) \equiv \lambda_{1}$, $s(t)=\lambda_{2}$ for all $t \in[0, T]$, then system (1.2) is reduced to

$$
\begin{cases}D^{\alpha_{2}}\left[D^{\alpha_{1}}+\lambda_{1}\right] x(t)=f(t, x(t), y(t)), & 0<t<T, \\ D^{\beta_{2}}\left[D^{\beta_{1}}+\lambda_{2}\right] y(t)=g(t, x(t), y(t)), & 0<t<T,\end{cases}
$$

which are Langevin fractional differential equations.

The paper is organized as follows. In Section 2, we recall definitions from fractional calculus and present an auxiliary lemma. The main results for the coupled system of generalized Sturm-Liouville and Langevin fractional differential equations with anti-periodic boundary conditions are discussed in Section 3. We give an existence and uniqueness result with the help of Banach's contraction mapping principle and an existence result via the Leray-Schauder alternative. Our results are well illustrated with the aid of examples presented in Section 4.

\section{Preliminaries}

In this section, we introduce some notations and definitions of fractional calculus (see [2]) and present preliminary results needed in our proofs later.

Definition 2.1 For an $(n-1)$-times absolutely continuous function $f:[0, \infty) \rightarrow \mathbb{R}$, the Caputo derivative of fractional order $\alpha>0$ is defined as

$$
D^{\alpha} f(t)=\frac{1}{\Gamma(n-\alpha)} \int_{0}^{t}(t-s)^{n-\alpha-1} f^{(n)}(s) d s, \quad n-1<\alpha<n,
$$

where $n=[\alpha]+1,[\alpha]$ denotes the integer part of the positive real number $\alpha$, and $\Gamma(\cdot)$ is the gamma function. 
Definition 2.2 The Riemann-Liouville fractional integral of order $\alpha$ of a function $f$ : $[0, \infty) \rightarrow \mathbb{R}$ is defined as

$$
I^{\alpha} f(t)=\frac{1}{\Gamma(\alpha)} \int_{0}^{t} \frac{f(s)}{(t-s)^{1-\alpha}} d s, \quad \alpha>0
$$

provided the integral exists.

Lemma 2.1 For $\alpha>0$, the general solution of the fractional differential equation $D^{\alpha} x(t)=0$ is given by

$$
x(t)=c_{0}+c_{1} t+\cdots+c_{n-1} t^{n-1},
$$

where $c_{i} \in \mathbb{R}, i=0,1,2, \ldots, n-1(n=[\alpha]+1)$.

In view of Lemma 2.1, it follows that

$$
I^{\alpha} D^{\alpha} x(t)=x(t)+c_{0}+c_{1} t+\cdots+c_{n-1} t^{n-1}
$$

for some $c_{i} \in \mathbb{R}, i=0,1,2, \ldots, n-1$.

Lemma 2.2 Let $u, v \in C([0, T], \mathbb{R})$ be two given functions. Then the following linear system of fractional differential equations subject to anti-periodic boundary conditions

$$
\left\{\begin{array}{l}
D^{\alpha_{2}}\left(\left[p(t) D^{\alpha_{1}}+r(t)\right] x(t)\right)=u(t), \quad 0<t<T, \\
D^{\beta_{2}}\left(\left[q(t) D^{\beta_{1}}+s(t)\right] y(t)\right)=v(t), \quad 0<t<T, \\
x(0)=-x(T), \quad D^{\alpha_{1}} x(0)=-D^{\alpha_{1}} x(T), \\
y(0)=-y(T), \quad D^{\beta_{1}} y(0)=-D^{\beta_{1}} y(T),
\end{array}\right.
$$

is equivalent to the following integral equations:

$$
\begin{aligned}
x(t)= & I^{\alpha_{1}}\left(\frac{1}{p} I^{\alpha_{2}} u\right)(t)-I^{\alpha_{1}}\left(\frac{r}{p} x\right)(t) \\
& +\left(\frac{-\gamma_{1}}{\gamma_{1}+1} I^{\alpha_{2}} u(T)+\frac{\eta_{1}}{\gamma_{1}+1} x(T)\right) I^{\alpha_{1}}\left(\frac{1}{p}\right)(t) \\
& -\frac{1}{2}\left[I^{\alpha_{1}}\left(\frac{1}{p} I^{\alpha_{2}} u\right)(T)-I^{\alpha_{1}}\left(\frac{r}{p} x\right)(T)\right. \\
& \left.+\left(\frac{-\gamma_{1}}{\gamma_{1}+1} I^{\alpha_{2}} u(T)+\frac{\eta_{1}}{\gamma_{1}+1} x(T)\right) \rho_{1}\right]
\end{aligned}
$$

and

$$
\begin{aligned}
y(t)= & I^{\beta_{1}}\left(\frac{1}{q} I^{\beta_{2}} v\right)(t)-I^{\beta_{1}}\left(\frac{s}{q} y\right)(t) \\
& +\left(\frac{-\gamma_{2}}{\gamma_{2}+1} I^{\beta_{2}} v(T)+\frac{\eta_{2}}{\gamma_{2}+1} y(T)\right) I^{\beta_{1}}\left(\frac{1}{q}\right)(t)
\end{aligned}
$$




$$
\begin{aligned}
& -\frac{1}{2}\left[I^{\beta_{1}}\left(\frac{1}{q} I^{\beta_{2}} v\right)(T)-I^{\beta_{1}}\left(\frac{s}{q} y\right)(T)\right. \\
& \left.+\left(\frac{-\gamma_{2}}{\gamma_{2}+1} I^{\beta_{2}} v(T)+\frac{\eta_{2}}{\gamma_{2}+1} y(T)\right) \rho_{2}\right],
\end{aligned}
$$

where

$$
\begin{aligned}
& \gamma_{1}=\frac{p(0)}{p(T)}, \quad \gamma_{2}=\frac{q(0)}{q(T)}, \quad \eta_{1}=\gamma_{1} r(T)-r(0), \quad \eta_{2}=\gamma_{2} s(T)-s(0), \\
& \rho_{1}=I^{\alpha_{1}}\left(\frac{1}{p}\right)(T), \quad \rho_{2}=I^{\beta_{1}}\left(\frac{1}{q}\right)(T) .
\end{aligned}
$$

Proof Taking the Riemann-Liouville fractional integral of orders $\alpha_{2}, \beta_{2}$ into the first two equations of system (2.4), we have

$$
\begin{aligned}
& D^{\alpha_{1}} x(t)=\frac{I^{\alpha_{2}} u(t)-r(t) x(t)+c_{0}}{p(t)}, \\
& D^{\beta_{1}} y(t)=\frac{I^{\beta_{2}} v(t)-s(t) y(t)+k_{0}}{q(t)},
\end{aligned}
$$

where $c_{0}, k_{0} \in \mathbb{R}$. From the boundary conditions of (2.4), we obtain

$$
c_{0}=\frac{-\gamma_{1}}{\gamma_{1}+1} I^{\alpha_{2}} u(T)+\frac{\eta_{1}}{\gamma_{1}+1} x(T)
$$

and

$$
k_{0}=\frac{-\gamma_{2}}{\gamma_{2}+1} I^{\beta_{2}} v(T)+\frac{\eta_{2}}{\gamma_{2}+1} y(T)
$$

Taking the Riemann-Liouville fractional integral of orders $\alpha_{1}, \beta_{1}$ into (2.7), (2.8), respectively, we get

$$
x(t)=I^{\alpha_{1}}\left(\frac{1}{p} I^{\alpha_{2}} u\right)(t)-I^{\alpha_{1}}\left(\frac{r}{p} x\right)(t)+c_{0} I^{\alpha_{1}}\left(\frac{1}{p}\right)(t)+c_{1}
$$

and

$$
y(t)=I^{\beta_{1}}\left(\frac{1}{q} I^{\beta_{2}} v\right)(t)-I^{\beta_{1}}\left(\frac{s}{q} y\right)(t)+k_{0} I^{\beta_{1}}\left(\frac{1}{q}\right)(t)+k_{1},
$$

where $c_{1}, k_{1} \in \mathbb{R}$. Using the boundary conditions of (2.4), we have

$$
c_{1}=-\frac{1}{2}\left[I^{\alpha_{1}}\left(\frac{1}{p} I^{\alpha_{2}} u\right)(T)-I^{\alpha_{1}}\left(\frac{r}{p} x\right)(T)+\left(\frac{-\gamma_{1}}{\gamma_{1}+1} I^{\alpha_{2}} u(T)+\frac{\eta_{1}}{\gamma_{1}+1} x(T)\right) \rho_{1}\right]
$$

and

$$
k_{1}=-\frac{1}{2}\left[I^{\beta_{1}}\left(\frac{1}{q} I^{\beta_{2}} v\right)(T)-I^{\beta_{1}}\left(\frac{s}{q} y\right)(T)+\left(\frac{-\gamma_{2}}{\gamma_{2}+1} I^{\beta_{2}} v(T)+\frac{\eta_{2}}{\gamma_{2}+1} y(T)\right) \rho_{2}\right] .
$$

Substituting the values of constants $c_{0}, c_{1}, k_{0}$ and $k_{1}$ into (2.9) and (2.10), we obtain the integral equations (2.5) and (2.6), respectively. The converse follows by a direct computation. This completes the proof. 


\section{Main results}

Throughout this paper, for convenience, we use the following expression:

$$
I^{\phi} h(s, x(s), y(s))(i)=\frac{1}{\Gamma(\phi)} \int_{0}^{i} \frac{h(s, x(s), y(s))}{(i-s)^{1-\phi}} d s
$$

where $\phi \in\left\{\alpha_{1}, \alpha_{2}, \beta_{1} \beta_{2}\right\}, i \in\{t, T\}, h=\{f, g\}$. Let us introduce the space $X=\{x(t) \mid x(t) \in$ $C([0, T], \mathbb{R})\}$ endowed with the norm $\|x\|=\sup \{|x(t)|, t \in[0, T]\}$. It is obvious that $(X,\|\cdot\|)$ is a Banach space. In addition, the product space $(X \times X,\|(x, y)\|)$ is a Banach space with the norm $\|(x, y)\|=\|x\|+\|y\|$. In view of Lemma (2.2), we define an operator $\mathcal{A}: X \times X \rightarrow$ $X \times X$ by

$$
\mathcal{A}(x, y)(t)=\left(\begin{array}{l}
\mathcal{A}_{1}(x, y)(t) \\
\mathcal{A}_{2}(x, y)(t)
\end{array}\right),
$$

where

$$
\begin{aligned}
\mathcal{A}_{1}(x, y)(t)= & I^{\alpha_{1}}\left(\frac{1}{p} I^{\alpha_{2}} f(s, x(s), y(s))\right)(t)-I^{\alpha_{1}}\left(\frac{r}{p} x\right)(t) \\
& +\left(\frac{-\gamma_{1}}{\gamma_{1}+1} I^{\alpha_{2}} f(s, x(s), y(s))(T)+\frac{\eta_{1}}{\gamma_{1}+1} x(T)\right) I^{\alpha_{1}}\left(\frac{1}{p}\right)(t) \\
& -\frac{1}{2}\left[I^{\alpha_{1}}\left(\frac{1}{p} I^{\alpha_{2}} f(s, x(s), y(s))\right)(T)-I^{\alpha_{1}}\left(\frac{r}{p} x\right)(T)\right. \\
& \left.+\left(\frac{-\gamma_{1}}{\gamma_{1}+1} I^{\alpha_{2}} f(s, x(s), y(s))(T)+\frac{\eta_{1}}{\gamma_{1}+1} x(T)\right) \rho_{1}\right]
\end{aligned}
$$

and

$$
\begin{aligned}
\mathcal{A}_{2}(x, y)(t)= & I^{\beta_{1}}\left(\frac{1}{q} I^{\beta_{2}} g(s, x(s), y(s))\right)(t)-I^{\beta_{1}}\left(\frac{s}{q} y\right)(t) \\
& +\left(\frac{-\gamma_{2}}{\gamma_{2}+1} I^{\beta_{2}} g(s, x(s), y(s))(T)+\frac{\eta_{2}}{\gamma_{2}+1} y(T)\right) I^{\beta_{1}}\left(\frac{1}{q}\right)(t) \\
& -\frac{1}{2}\left[I^{\beta_{1}}\left(\frac{1}{q} I^{\beta_{2}} g(s, x(s), y(s))\right)(T)-I^{\beta_{1}}\left(\frac{s}{q} y\right)(T)\right. \\
& \left.+\left(\frac{-\gamma_{2}}{\gamma_{2}+1} I^{\beta_{2}} g(s, x(s), y(s))(T)+\frac{\eta_{2}}{\gamma_{2}+1} y(T)\right) \rho_{2}\right] .
\end{aligned}
$$

We set the following constants:

$$
p^{*}=\inf _{t \in[0, T]}|p(t)|, \quad q^{*}=\inf _{t \in[0, T]}|q(t)|, \quad r^{*}=\sup _{t \in[0, T]}|r(t)|, \quad s^{*}=\sup _{t \in[0, T]}|s(t)|
$$

and

$$
\begin{aligned}
& h_{1}=\frac{T^{\alpha_{1}+\alpha_{2}}}{p^{*} \Gamma\left(1+\alpha_{1}+\alpha_{2}\right)}, \quad h_{2}=\frac{r^{*} T^{\alpha_{1}}}{p^{*} \Gamma\left(1+\alpha_{1}\right)}, \\
& h_{3}=\frac{\gamma_{1} T^{\alpha_{1}+\alpha_{2}}}{p^{*}\left(\gamma_{1}+1\right) \Gamma\left(1+\alpha_{1}\right) \Gamma\left(1+\alpha_{2}\right)}, \quad h_{4}=\frac{\left|\eta_{1}\right| T^{\alpha_{1}}}{p^{*}\left(\gamma_{1}+1\right) \Gamma\left(1+\alpha_{1}\right)},
\end{aligned}
$$




$$
\begin{aligned}
& h_{5}=\frac{T^{\beta_{1}+\beta_{2}}}{q^{*} \Gamma\left(1+\beta_{1}+\beta_{2}\right)}, \quad h_{6}=\frac{s^{*} T^{\beta_{1}}}{q^{*} \Gamma\left(1+\beta_{1}\right)}, \\
& h_{7}=\frac{\gamma_{2} T^{\beta_{1}+\beta_{2}}}{q^{*}\left(\gamma_{2}+1\right) \Gamma\left(1+\beta_{1}\right) \Gamma\left(1+\beta_{2}\right)}, \quad h_{8}=\frac{\left|\eta_{2}\right| T^{\beta_{1}}}{q^{*}\left(\gamma_{2}+1\right) \Gamma\left(1+\beta_{1}\right)} .
\end{aligned}
$$

Theorem 3.1 Assume that $f, g:[0, T] \times \mathbb{R}^{2} \rightarrow \mathbb{R}$ are continuous functions, and there exist constants $m_{i}, n_{i}, i=1,2$ such that for all $t \in[0, T]$ and $x_{i}, y_{i} \in \mathbb{R}, i=1,2$,

$\left(H_{1}\right)\left|f\left(t, x_{1}, y_{1}\right)-f\left(t, x_{2}, y_{2}\right)\right| \leq m_{1}\left|x_{1}-x_{2}\right|+m_{2}\left|y_{1}-y_{2}\right|$,

$\left(H_{2}\right)\left|g\left(t, x_{1}, y_{1}\right)-g\left(t, x_{2}, y_{2}\right)\right| \leq n_{1}\left|x_{1}-x_{2}\right|+n_{2}\left|y_{1}-y_{2}\right|$.

In addition, let

$$
L_{1}+L_{2}<1,
$$

where

$$
\begin{aligned}
& M_{1}=\frac{3}{2}\left(h_{1}+h_{3}\right), \quad M_{2}=\frac{3}{2}\left(h_{2}+h_{4}\right), \quad M_{3}=\frac{3}{2}\left(h_{5}+h_{7}\right), \quad M_{4}=\frac{3}{2}\left(h_{6}+h_{8}\right), \\
& L_{1}=\left(m_{1}+m_{2}\right) M_{1}+M_{2}, \quad L_{2}=\left(n_{1}+n_{2}\right) M_{3}+M_{4} .
\end{aligned}
$$

Then problem (1.2)-(1.3) has a unique solution on $[0, T]$.

Proof To show that problem (1.2)-(1.3) has a unique solution on [0, T], we will use Banach's contraction mapping principle. In the first step, we define $\sup _{t \in[0, T]} f(t, 0,0)=N_{1}<\infty$, $\sup _{t \in[0, T]} g(t, 0,0)=N_{2}<\infty$ and choose a positive real number $w$ such that

$$
w \geq \frac{N_{1} M_{1}+N_{2} M_{2}}{1-L_{1}-L_{2}} .
$$

Now, we show that $\mathcal{A} B_{w} \subset B_{w}$, where $B_{w}=\{(x, y) \in X \times X:\|(x, y)\| \leq w\}$. For any $(x, y) \in B_{w}$, we have

$$
\begin{aligned}
\left|\mathcal{A}_{1}(x, y)(t)\right| & \\
\leq & \sup _{t \in[0, T]}\left\{I^{\alpha_{1}}\left(\frac{1}{p^{*}} I^{\alpha_{2}}|f(s, x(s), y(s))|\right)(t)+I^{\alpha_{1}}\left(\frac{r^{*}}{p^{*}}|x(s)|\right)(t)\right. \\
& +\left(\frac{\gamma_{1}}{\gamma_{1}+1} I^{\alpha_{2}}|f(s, x(s), y(s))|(T)+\frac{\left|\eta_{1}\right|}{\gamma_{1}+1}|x(T)|\right)\left|I^{\alpha_{1}}\left(\frac{1}{p}\right)(t)\right| \\
& +\frac{1}{2}\left[I^{\alpha_{1}}\left(\frac{1}{p^{*}} I^{\alpha_{2}}|f(s, x(s), y(s))|\right)(T)+I^{\alpha_{1}}\left(\frac{r^{*}}{p^{*}}|x(s)|\right)(T)\right. \\
& \left.\left.+\left(\frac{\gamma_{1}}{\gamma_{1}+1} I^{\alpha_{2}}|f(s, x(s), y(s))|(T)+\frac{\left|\eta_{1}\right|}{\gamma_{1}+1}|x(T)|\right)\left|\rho_{1}\right|\right]\right\} \\
\leq & I^{\alpha_{1}}\left(\frac{1}{p^{*}} I^{\alpha_{2}}|f(s, x(s), y(s))-f(s, 0,0)|+|f(s, 0,0)|\right)(T) \\
& +I^{\alpha_{1}}\left(\frac{r^{*}}{p^{*}}|x(s)|\right)(T)+\left(\frac{\gamma_{1}}{\gamma_{1}+1} I^{\alpha_{2}}(|f(s, x(s), y(s))-f(s, 0,0)|\right. \\
& \left.+|f(s, 0,0)|)(T)+\frac{\left|\eta_{1}\right|}{\gamma_{1}+1}|x(T)|\right)\left|I^{\alpha_{1}}\left(\frac{1}{p}\right)(T)\right|
\end{aligned}
$$




$$
\begin{aligned}
& +\frac{1}{2}\left[I^{\alpha_{1}}\left(\frac{1}{p^{*}} I^{\alpha_{2}}|f(s, x(s), y(s))-f(s, 0,0)|+|f(s, 0,0)|\right)(T)\right. \\
& +I^{\alpha_{1}}\left(\frac{r^{*}}{p^{*}}|x(s)|\right)(T)+\left(\frac{\gamma_{1}}{\gamma_{1}+1} I^{\alpha_{2}}(|f(s, x(s), y(s))-f(s, 0,0)|\right. \\
& \left.\left.+|f(s, 0,0)|)(T)+\frac{\left|\eta_{1}\right|}{\gamma_{1}+1}|x(T)|\right)\left|\rho_{1}\right|\right] \\
& \leq I^{\alpha_{1}}\left(\left(m_{1}\|x\|+m_{2}\|y\|+N_{1}\right) \frac{1}{p^{*}} I^{\alpha_{2}}(1)\right)(T)+\left(\frac{r^{*}}{p^{*}}\right)\|x\| I^{\alpha_{1}}(1)(T) \\
& +\left(\frac{\gamma_{1}}{\gamma_{1}+1}\left(m_{1}\|x\|+m_{2}\|y\|+N_{1}\right) I^{\alpha_{2}}(1)(T)+\frac{\left|\eta_{1}\right|}{\gamma_{1}+1}\|x\|\right)\left|I^{\alpha_{1}}\left(\frac{1}{p}\right)(T)\right| \\
& +\frac{1}{2}\left[I^{\alpha_{1}}\left(\left(m_{1}\|x\|+m_{2}\|y\|+N_{1}\right) \frac{1}{p^{*}} I^{\alpha_{2}}(1)\right)(T)+\left(\frac{r^{*}}{p^{*}}\|x\|\right) I^{\alpha_{1}}(1)(T)\right. \\
& \left.+\left(\frac{\gamma_{1}}{\gamma_{1}+1}\left(m_{1}\|x\|+m_{2}\|y\|+N_{1}\right) I^{\alpha_{2}}(1)(T)+\frac{\left|\eta_{1}\right|}{\gamma_{1}+1}\|x\|\right)\left|\rho_{1}\right|\right] \\
& \leq\left(m_{1}\|x\|+m_{2}\|y\|+N_{1}\right) \frac{T^{\alpha_{1}+\alpha_{2}}}{p^{*} \Gamma\left(1+\alpha_{1}+\alpha_{2}\right)}+\frac{r^{*} T^{\alpha_{1}}}{p^{*} \Gamma\left(1+\alpha_{1}\right)}\|x\| \\
& +\left(m_{1}\|x\|+m_{2}\|y\|+N_{1}\right) \frac{\gamma_{1} T^{\alpha_{1}+\alpha_{2}}}{p^{*}\left(\gamma_{1}+1\right) \Gamma\left(1+\alpha_{1}\right) \Gamma\left(1+\alpha_{2}\right)} \\
& +\frac{\left|\eta_{1}\right| T^{\alpha_{1}}}{p^{*}\left(\gamma_{1}+1\right) \Gamma\left(1+\alpha_{1}\right)}\|x\|+\frac{1}{2} \frac{\left|\eta_{1}\right| T^{\alpha_{1}}}{p^{*}\left(\gamma_{1}+1\right) \Gamma\left(1+\alpha_{1}\right)}\|x\| \\
& +\frac{1}{2}\left(m_{1}\|x\|+m_{2}\|y\|+N_{1}\right) \frac{T^{\alpha_{1}+\alpha_{2}}}{p^{*} \Gamma\left(1+\alpha_{1}+\alpha_{2}\right)}+\frac{1}{2} \frac{r^{*} T^{\alpha_{1}}}{p^{*} \Gamma\left(1+\alpha_{1}\right)}\|x\| \\
& +\frac{1}{2} \frac{\gamma_{1} T^{\alpha_{1}+\alpha_{2}}}{p^{*}\left(\gamma_{1}+1\right) \Gamma\left(1+\alpha_{1}\right) \Gamma\left(1+\alpha_{2}\right)}\left(m_{1}\|x\|+m_{2}\|y\|+N_{1}\right) \\
& =\frac{3}{2}\left(m_{1}\|x\|+m_{2}\|y\|+N_{1}\right)\left(h_{1}+h_{3}\right)+\frac{3}{2}\left(h_{2}+h_{4}\right)\|x\| \\
& =\left(m_{1} M_{1}+M_{2}\right)\|x\|+m_{2} M_{1}\|y\|+N_{1} M_{1} \\
& \leq\left(m_{1} M_{1}+M_{2}\right) w+m_{2} M_{1} w+N_{1} M_{1} \\
& =L_{1} w+N_{1} M_{1} \text {. }
\end{aligned}
$$

Similarly, we obtain

$$
\begin{aligned}
\left|\mathcal{A}_{2}(x, y)(t)\right| & \\
\leq & \left(n_{1}\|x\|+n_{2}\|y\|+N_{2}\right) \frac{T^{\beta_{1}+\beta_{2}}}{q^{*} \Gamma\left(1+\beta_{1}+\beta_{2}\right)}+\frac{s^{*} T^{\beta_{1}}}{q^{*} \Gamma\left(1+\beta_{1}\right)}\|y\| \\
& +\left(n_{1}\|x\|+n_{2}\|y\|+N_{2}\right) \frac{\gamma_{2} T^{\beta_{1}+\beta_{2}}}{q^{*}\left(\gamma_{2}+1\right) \Gamma\left(1+\beta_{1}\right) \Gamma\left(1+\beta_{2}\right)} \\
& +\frac{\left|\eta_{2}\right| T^{\beta_{1}}}{q^{*}\left(\gamma_{2}+1\right) \Gamma\left(1+\beta_{1}\right)}\|y\|+\frac{1}{2} \frac{\left|\eta_{2}\right| T^{\beta_{1}}}{q^{*}\left(\gamma_{2}+1\right) \Gamma\left(1+\beta_{1}\right)}\|y\| \\
& +\frac{1}{2}\left(n_{1}\|x\|+n_{2}\|y\|+N_{2}\right) \frac{T^{\beta_{1}+\beta_{2}}}{q^{*} \Gamma\left(1+\beta_{1}+\beta_{2}\right)}+\frac{1}{2} \frac{s^{*} T^{\beta_{1}}}{q^{*} \Gamma\left(1+\beta_{1}\right)}\|y\| \\
& +\frac{1}{2} \frac{\gamma_{2} T^{\beta_{1}+\beta_{2}}}{q^{*}\left(\gamma_{2}+1\right) \Gamma\left(1+\beta_{1}\right) \Gamma\left(1+\beta_{2}\right)}\left(n_{1}\|x\|+n_{2}\|y\|+N_{2}\right)
\end{aligned}
$$




$$
\begin{aligned}
& =\frac{3}{2}\left(n_{1}\|x\|+n_{2}\|y\|+N_{2}\right)\left(h_{5}+h_{7}\right)+\frac{3}{2}\left(h_{6}+h_{8}\right)\|y\| \\
& =n_{1} M_{3}\|x\|+\left(n_{2} M_{3}+M_{4}\right)\|y\|+N_{2} M_{3} \\
& \leq n_{1} M_{3} w+\left(n_{2} M_{3}+M_{4}\right) w+N_{2} M_{3} \\
& =L_{2} w+N_{2} M_{3} .
\end{aligned}
$$

Therefore, we deduce that

$$
\|\mathcal{A}(x, y)\|=\left\|\mathcal{A}_{1}(x, y)\right\|+\left\|\mathcal{A}_{2}(x, y)\right\| \leq\left(L_{1}+L_{2}\right) w+N_{1} M_{1}+N_{2} M_{3}<w,
$$

which implies $\mathcal{A} B_{w} \subset B_{w}$.

Next, for $\left(x_{2}, y_{2}\right),\left(x_{1}, y_{1}\right) \in X \times X$ and for any $t \in[0, T]$, we have

$$
\begin{aligned}
& \left|\mathcal{A}_{1}\left(x_{2}, y_{2}\right)(t)-\mathcal{A}_{1}\left(x_{1}, y_{1}\right)(t)\right| \\
& \leq I^{\alpha_{1}}\left(\frac{1}{p} I^{\alpha_{2}}\left|f\left(s, x_{2}(s), y_{2}(s)\right)-f\left(s, x_{1}(s), y_{1}(s)\right)\right|\right)(T) \\
& +I^{\alpha_{1}}\left(\frac{r^{*}}{p^{*}}\left|x_{2}(s)-x_{1}(s)\right|\right)(T) \\
& +\left(\frac { \gamma _ { 1 } } { \gamma _ { 1 } + 1 } I ^ { \alpha _ { 2 } } \left(\left|f\left(s, x_{2}(s), y_{2}(s)\right)-f\left(s, x_{1}(s), y_{1}(s)\right)\right|(T)\right.\right. \\
& \left.+\frac{\left|\eta_{1}\right|}{\gamma_{1}+1}\left|x_{2}(T)-x_{1}(T)\right|\right)\left|I^{\alpha_{1}}\left(\frac{1}{p}\right)(T)\right| \\
& +\frac{1}{2}\left[I^{\alpha_{1}}\left(\frac{1}{p} I^{\alpha_{2}}\left|f\left(s, x_{2}(s), y_{2}(s)\right)-f\left(s, x_{1}(s), y_{1}(s)\right)\right|\right)(T)\right. \\
& +I^{\alpha_{1}}\left(\frac{r^{*}}{p}\left|x_{2}(s)-x_{1}(s)\right|\right)(T)+\left(\frac { \gamma _ { 1 } } { \gamma _ { 1 } + 1 } I ^ { \alpha _ { 2 } } \left(\mid f\left(s, x_{2}(s), y_{2}(s)\right)\right.\right. \\
& \left.\left.-f\left(s, x_{1}(s), y_{1}(s)\right)\left|(T)+\frac{\left|\eta_{1}\right|}{\gamma_{1}+1}\right| x_{2}(T)-x_{1}(T) \mid\right)\left|\rho_{1}\right|\right] \\
& \leq \frac{T^{\alpha_{1}+\alpha_{2}}}{p^{*} \Gamma\left(1+\alpha_{1}+\alpha_{2}\right)}\left(m_{1}\left\|x_{2}-x_{1}\right\|+m_{2}\left\|y_{2}-y_{1}\right\|\right)+\frac{r^{*} T^{\alpha_{1}}}{p^{*} \Gamma\left(1+\alpha_{1}\right)}\left\|x_{2}-x_{1}\right\| \\
& +\frac{\gamma_{1} T^{\alpha_{1}+\alpha_{2}}}{p^{*}\left(\gamma_{1}+1\right) \Gamma\left(1+\alpha_{1}\right) \Gamma\left(1+\alpha_{2}\right)}\left(m_{1}\left\|x_{2}-x_{1}\right\|+m_{2}\left\|y_{2}-y_{1}\right\|\right) \\
& +\frac{\left|\eta_{1}\right| T^{\alpha_{1}}}{p^{*}\left(\gamma_{1}+1\right) \Gamma\left(1+\alpha_{1}\right)}\left\|x_{2}-x_{1}\right\|+\frac{1}{2} \frac{\left|\eta_{1}\right| T^{\alpha_{1}}}{p^{*}\left(\gamma_{1}+1\right) \Gamma\left(1+\alpha_{1}\right)}\left\|x_{2}-x_{1}\right\| \\
& +\frac{1}{2} \frac{T^{\alpha_{1}+\alpha_{2}}}{p^{*} \Gamma\left(\alpha_{1}+\alpha_{2}\right)}\left(m_{1}\left\|x_{2}-x_{1}\right\|+m_{2}\left\|y_{2}-y_{1}\right\|\right)+\frac{1}{2} \frac{r^{*} T^{\alpha_{1}}}{p^{*} \Gamma\left(\alpha_{1}\right)}\left\|x_{2}-x_{1}\right\| \\
& +\frac{1}{2} \frac{\gamma_{1} T^{1+\alpha_{1}+\alpha_{2}}}{p^{*}\left(\gamma_{1}+1\right) \Gamma\left(\alpha_{1}\right) \Gamma\left(\alpha_{2}\right)}\left(m_{1}\left\|x_{2}-x_{1}\right\|+m_{2}\left\|y_{2}-y_{1}\right\|\right) \\
& =\frac{3}{2}\left(h_{1}+h_{3}\right)\left(m_{1}\left\|x_{2}-x_{1}\right\|+m_{2}\left\|y_{2}-y_{1}\right\|\right)+\frac{3}{2}\left(h_{2}+h_{4}\right)\left\|x_{2}-x_{1}\right\| \\
& =\left(m_{1} M_{1}+M_{2}\right)\left\|x_{2}-x_{1}\right\|+m_{2} M_{1}\left\|y_{2}-y_{1}\right\| \text {, }
\end{aligned}
$$


which leads to

$$
\left\|\mathcal{A}_{1}\left(x_{2}, y_{2}\right)-\mathcal{A}_{1}\left(x_{1}, y_{1}\right)\right\| \leq L_{1}\left(\left\|x_{2}-x_{1}\right\|+\left\|y_{2}-y_{1}\right\|\right)
$$

Similarly, we obtain

$$
\left\|\mathcal{A}_{2}\left(x_{2}, y_{2}\right)-\mathcal{A}_{2}\left(x_{1}, y_{1}\right)\right\| \leq L_{2}\left(\left\|x_{2}-x_{1}\right\|+\left\|y_{2}-y_{1}\right\|\right) .
$$

Hence, from (3.1) and (3.2), we deduce that

$$
\left\|\mathcal{A}\left(x_{2}, y_{2}\right)-\mathcal{A}\left(x_{1}, y_{1}\right)\right\| \leq\left(L_{1}+L_{2}\right)\left(\left\|x_{2}-x_{1}\right\|+\left\|y_{2}-y_{1}\right\|\right) .
$$

Since $L_{1}+L_{2}<1, \mathcal{A}$ is a contraction operator. Thus, by Banach's fixed point theorem, the operator $\mathcal{A}$ has a unique fixed point, which is the unique solution of problem (1.2)-(1.3) on $[0, T]$. This completes the proof.

If $r(t), s(t) \equiv 0$ for all $t \in[0, T]$, then we have $\eta_{1}, \eta_{2}=0$.

Corollary 3.1 Suppose that conditions $\left(H_{1}\right)-\left(H_{2}\right)$ hold. If $\left(m_{1}+m_{2}\right) M_{1}+\left(n_{1}+n_{2}\right) M_{3}<1$, then system (1.4) with (1.3) has a unique solution on $[0, T]$.

$$
\begin{aligned}
& \text { If } p(t), q(t) \equiv 1 \text { and } r(t) \equiv \lambda_{1}, s(t)=\lambda_{2} \text { for all } t \in[0, T], \text { then we get that } p^{*}, q^{*}=1, r^{*}=\left|\lambda_{1}\right|, \\
& s^{*}=\left|\lambda_{2}\right|, \gamma_{1}, \gamma_{2}=1, \eta_{1}, \eta_{2}=0 . \text { Let } \\
& a_{1}=\frac{3}{2} \frac{T^{\alpha_{1}+\alpha_{2}}}{\Gamma\left(1+\alpha_{1}+\alpha_{2}\right)}+\frac{3}{4} \frac{T^{\alpha_{1}+\alpha_{2}}}{\Gamma\left(1+\alpha_{1}\right) \Gamma\left(1+\alpha_{2}\right)}, \\
& a_{2}=\frac{3}{2}\left|\lambda_{1}\right| \frac{T^{\alpha_{1}}}{\Gamma\left(1+\alpha_{1}\right)}, \\
& a_{3}=\frac{3}{2} \frac{T^{\beta_{1}+\beta_{2}}}{\Gamma\left(1+\beta_{1}+\beta_{2}\right)}+\frac{3}{4} \frac{T^{\beta_{1}+\beta_{2}}}{\Gamma\left(1+\beta_{1}\right) \Gamma\left(1+\beta_{2}\right)}, \\
& a_{4}=\frac{3}{2}\left|\lambda_{2}\right| \frac{T^{\beta_{1}}}{\Gamma\left(1+\beta_{1}\right)} .
\end{aligned}
$$

Corollary 3.2 Assume that conditions $\left(H_{1}\right)-\left(H_{2}\right)$ are satisfied. If

$$
\left[a_{1}\left(m_{1}+m_{2}\right)+a_{2}\right]+\left[a_{3}\left(n_{1}+n_{2}\right)+a_{4}\right]<1
$$

then system (1.5) with (1.3) has a unique solution on $[0, T]$.

In the next result, we will show the existence of solutions of problem (1.2)-(1.3) by applying the Leray-Schauder alternative.

Lemma 3.1 (Leray-Schauder alternative [34]) Let $G$ be a normed linear space and $F: G \rightarrow$ $G$ be a completely continuous operator (i.e., a map restricted to any bounded set in $G$ is compact). Let

$$
\mathcal{J}(F)=\{x \in G: x=\kappa F(x) \text { for some } 0<\kappa<1\} .
$$

Then either the set $\mathcal{J}(F)$ is unbounded, or $F$ has at least one fixed point. 
Theorem 3.2 Assume that $f, g:(0, T) \times \mathbb{R}^{2} \rightarrow \mathbb{R}$ are two continuous functions and there exist real constants $C_{i}, D_{i} \geq 0(i=1,2)$ and $C_{0}, D_{0}>0$ such that $\forall x_{i}, y_{i} \in \mathbb{R},(i=1,2)$ satisfying

( $\left.H_{3}\right) \quad\left|f\left(t, x_{1}, x_{2}\right)\right| \leq C_{0}+C_{1}\left|x_{1}\right|+C_{2}\left|x_{2}\right|$ and

$\left(H_{4}\right)\left|g\left(t, y_{1}, y_{2}\right)\right| \leq D_{0}+D_{1}\left|y_{1}\right|+D_{2}\left|y_{2}\right|$.

In addition, it is assumed that

$$
J_{1}<1 \text { and } J_{2}<1 \text {, }
$$

where

$$
J_{1}=1-\left(C_{1} M_{1}+M_{2}+D_{1} M_{3}\right), \text { and } J_{2}=1-\left(C_{2} M_{1}+D_{2} M_{3}+M_{4}\right) .
$$

Then there exists at least one solution of problem (1.2)-(1.3) on $[0, T]$.

Proof Firstly, we show that the operator $\mathcal{A}: X \times X \rightarrow X \times X$ is completely continuous. Note that $\mathcal{A}$ is continuous, since the functions $f, g$ are continuous. Let $U \subset X \times X$ be a bounded set. Then there exists a positive constant $\hat{w}$ such that $\|(x, y)\| \leq \hat{w}$ for any $(x, y) \in U$. Also there exist $S_{1}$ and $S_{2}$ such that

$$
|f(t, x(t), y(t))| \leq S_{1}, \quad|g(t, x(t), y(t))| \leq S_{2}, \quad \forall(x, y) \in U .
$$

Therefore, for any $(x, y) \in U$, we have

$$
\begin{aligned}
& \left|\mathcal{A}_{1}(x, y)(t)\right| \\
& \leq I^{\alpha_{1}}\left(\frac{1}{p} I^{\alpha_{2}}|f(s, x(s), y(s))|\right)(T)+I^{\alpha_{1}}\left(\frac{r^{*}}{p^{*}}|x(s)|\right)(T) \\
& +\left(\frac{\gamma_{1}}{\gamma_{1}+1} I^{\alpha_{2}}|f(s, x(s), y(s))|(T)+\frac{\left|\eta_{1}\right|}{\gamma_{1}+1}|x(T)|\right)\left|I^{\alpha_{1}}\left(\frac{1}{p}\right)(T)\right| \\
& +\frac{1}{2}\left[I^{\alpha_{1}}\left(\frac{1}{p} I^{\alpha_{2}}|f(s, x(s), y(s))|\right)(T)+I^{\alpha_{1}}\left(\frac{r^{*}}{p^{*}}|x(s)|\right)(T)\right. \\
& \left.+\left(\frac{\gamma_{1}}{\gamma_{1}+1} I^{\alpha_{2}}|f(s, x(s), y(s))|(T)+\frac{\left|\eta_{1}\right|}{\gamma_{1}+1}|x(T)|\right)\left|\rho_{1}\right|\right] \\
& \leq \frac{T^{\alpha_{1}+\alpha_{2}}}{p^{*} \Gamma\left(1+\alpha_{1}+\alpha_{2}\right)} S_{1}+\frac{r^{*} T^{\alpha_{1}}}{p^{*} \Gamma\left(1+\alpha_{1}\right)} \hat{w}+\frac{\gamma_{1} T^{\alpha_{1}+\alpha_{2}}}{p^{*}\left(\gamma_{1}+1\right) \Gamma\left(1+\alpha_{1}\right) \Gamma\left(1+\alpha_{2}\right)} S_{1} \\
& +\frac{\left|\eta_{1}\right| T^{\alpha_{1}}}{p^{*}\left(\gamma_{1}+1\right) \Gamma\left(1+\alpha_{1}\right)} \hat{w}+\frac{1}{2} \frac{T^{\alpha_{1}+\alpha_{2}}}{p^{*} \Gamma\left(1+\alpha_{1}+\alpha_{2}\right)} S_{1}+\frac{1}{2} \frac{r^{*} T^{\alpha_{1}}}{p^{*} \Gamma\left(1+\alpha_{1}\right)} \hat{w} \\
& +\frac{1}{2} \frac{\gamma_{1} T^{\alpha_{1}+\alpha_{2}}}{p^{*}\left(\gamma_{1}+1\right) \Gamma\left(1+\alpha_{1}\right) \Gamma\left(1+\alpha_{2}\right)} S_{1}+\frac{1}{2} \frac{\left|\eta_{1}\right| T^{\alpha_{1}}}{p^{*}\left(\gamma_{1}+1\right) \Gamma\left(1+\alpha_{1}\right)} \hat{w} \\
& =\frac{3}{2}\left(h_{1}+h_{3}\right) S_{1}+\frac{3}{2}\left(h_{2}+h_{4}\right) \hat{w} \\
& =M_{1} S_{1}+M_{2} \hat{w} \text {. }
\end{aligned}
$$

Thus $\left\|\mathcal{A}_{1}(x, y)\right\| \leq M_{1} S_{1}+M_{2} \hat{w}$. 
Similarly, we deduce that

$$
\begin{aligned}
\left|\mathcal{A}_{2}(x, y)(t)\right| \leq & \frac{T^{\beta_{1}+\beta_{2}}}{q^{*} \Gamma\left(1+\beta_{1}+\beta_{2}\right)} S_{2}+\frac{s^{*} T^{\beta_{1}}}{q^{*} \Gamma\left(1+\beta_{1}\right)} \hat{w} \\
& +\frac{\gamma_{2} T^{\beta_{1}+\beta_{2}}}{q^{*}\left(\gamma_{2}+1\right) \Gamma\left(1+\beta_{1}\right) \Gamma\left(1+\beta_{2}\right)} S_{2}+\frac{\left|\eta_{2}\right| T^{\beta_{1}}}{q^{*}\left(\gamma_{2}+1\right) \Gamma\left(1+\beta_{1}\right)} \hat{w} \\
& +\frac{1}{2} \frac{T^{\beta_{1}+\beta_{2}}}{q^{*} \Gamma\left(1+\beta_{1}+\beta_{2}\right)} S_{2}+\frac{1}{2} \frac{s^{*} T^{\beta_{1}}}{q^{*} \Gamma\left(1+\beta_{1}\right)} \hat{w} \\
& +\frac{1}{2} \frac{\gamma_{2} T^{\beta_{1}+\beta_{2}}}{q^{*}\left(\gamma_{2}+1\right) \Gamma\left(1+\beta_{1}\right) \Gamma\left(1+\beta_{2}\right)} S_{2}+\frac{1}{2} \frac{\left|\eta_{2}\right| T^{\beta_{1}}}{q^{*}\left(\gamma_{2}+1\right) \Gamma\left(1+\beta_{1}\right)} \hat{w} \\
= & \frac{3}{2}\left(h_{5}+h_{7}\right) S_{2}+\frac{3}{2}\left(h_{6}+h_{8}\right) \hat{w} \\
= & M_{3} S_{2}+M_{4} \hat{w},
\end{aligned}
$$

and therefore $\left\|\mathcal{A}_{2}(x, y)\right\| \leq M_{3} S_{2}+M_{4} \hat{w}$. Consequently, $\|\mathcal{A}(x, y)\| \leq M_{1} S_{1}+M_{3} S_{2}+\left(M_{2}+\right.$ $\left.M_{4}\right) \hat{w}$, which means that the operator $\mathcal{A}$ is uniformly bounded.

Next, we prove that $\mathcal{A}$ is equicontinuous. For given $t_{1}, t_{2} \in[0, T]$, with $t_{1}<t_{2}$, we get

$$
\begin{aligned}
& \left|\mathcal{A}_{1}(x, y)\left(t_{2}\right)-\mathcal{A}_{1}(x, y)\left(t_{1}\right)\right| \\
& \leq\left|I^{\alpha_{1}}\left(\frac{1}{p^{*}} I^{\alpha_{2}} f(s, x(s), y(s))\right)\left(t_{2}\right)-I^{\alpha_{1}}\left(\frac{1}{p^{*}} I^{\alpha_{2}} f(s, x(s), y(s))\right)\left(t_{1}\right)\right| \\
& +\left|I^{\alpha_{1}}\left(\frac{r^{*}}{p^{*}} x(s)\right)\left(t_{2}\right)-I^{\alpha_{1}}\left(\frac{r^{*}}{p^{*}} x(s)\right)\left(t_{1}\right)\right| \\
& +\left|\frac{\gamma_{1}}{\gamma_{1}+1} I^{\alpha_{2}} f(s, x(s), y(s))(T)+\frac{\eta_{1}}{\gamma_{1}+1} x(T)\right|\left|I^{\alpha_{1}}\left(\frac{1}{p}\right)\left(t_{2}\right)-I^{\alpha_{1}}\left(\frac{1}{p}\right)\left(t_{1}\right)\right| \\
& \leq \frac{S_{1}}{p^{*} \Gamma\left(\alpha_{1}+\alpha_{2}\right)}\left[\int_{0}^{t_{1}}\left|\left(t_{2}-s\right)^{\alpha_{1}+\alpha_{2}-1}-\left(t_{1}-s\right)^{\alpha_{1}+\alpha_{2}-1}\right| d s+\int_{t_{1}}^{t_{2}}\left(t_{2}-s\right)^{\alpha_{1}+\alpha_{2}-1} d s\right] \\
& +\frac{r^{*} \hat{w}}{p^{*} \Gamma\left(\alpha_{1}\right)}\left[\int_{0}^{t_{1}}\left|\left(t_{2}-s\right)^{\alpha_{1}-1}-\left(t_{1}-s\right)^{\alpha_{1}-1}\right| d s+\int_{t_{1}}^{t_{2}}\left(t_{2}-s\right)^{\alpha_{1}-1} d s\right] \\
& +\left(\frac{\gamma_{1} T^{\alpha_{2}} S_{1}}{\left(\gamma_{1}+1\right) \Gamma\left(1+\alpha_{2}\right)}+\frac{\left|\eta_{1}\right| \hat{w}}{\gamma_{1}+1}\right) \frac{1}{\Gamma\left(\alpha_{1}\right)}\left[\int_{0}^{t_{1}}\left|\left(t_{2}-s\right)^{\alpha_{1}-1}-\left(t_{1}-s\right)^{\alpha_{1}-1}\right| d s\right. \\
& \left.+\int_{t_{1}}^{t_{2}}\left(t_{2}-s\right)^{\alpha_{1}-1} d s\right] \\
& \leq \frac{S_{1}}{p^{*} \Gamma\left(1+\alpha_{1}+\alpha_{2}\right)}\left[2\left(t_{2}-t_{1}\right)^{\alpha_{1}+\alpha_{2}}+\left|t_{2}^{\alpha_{1}+\alpha_{2}}-t_{1}^{\alpha_{1}+\alpha_{2}}\right|\right] \\
& +\frac{1}{\Gamma\left(1+\alpha_{1}\right)}\left(\frac{r^{*} \hat{w}}{p^{*}}+\frac{\gamma_{1} T^{\alpha_{2}} S_{1}}{\left(\gamma_{1}+1\right) \Gamma\left(1+\alpha_{2}\right)}+\frac{\left|\eta_{1}\right| \hat{w}}{\gamma_{1}+1}\right)\left[2\left(t_{2}-t_{1}\right)^{\alpha_{1}}+\left|t_{2}^{\alpha_{1}}-t_{1}^{\alpha_{1}}\right|\right] .
\end{aligned}
$$

Hence we have

$$
\left\|\mathcal{A}_{1}(x, y)\left(t_{2}\right)-\mathcal{A}_{1}(x, y)\left(t_{1}\right)\right\| \rightarrow 0, \quad \text { as } t_{1} \rightarrow t_{2} .
$$

Analogously, we can obtain

$$
\left\|\mathcal{A}_{2}(x, y)\left(t_{2}\right)-\mathcal{A}_{2}(x, y)\left(t_{1}\right)\right\| \rightarrow 0, \quad \text { as } t_{1} \rightarrow t_{2} .
$$


Therefore, the operator $\mathcal{A}$ is equicontinuous, and thus the operator $\mathcal{A}$ is completely continuous.

Finally, it will be verified that the set

$$
\mathcal{J}=\{(x, y) \in X \times X \mid(x, y)=\kappa \mathcal{A}(x, y) \text {, for some } 0<\kappa<1\}
$$

is bounded. Let $(x, y) \in \mathcal{J}$, then $(x, y)=\kappa \mathcal{A}(x, y)$. For any $t \in[0, T]$, we have

$$
x(t)=\kappa \mathcal{A}_{1}(x, y)(t), \quad y(t)=\kappa \mathcal{A}_{2}(x, y)(t) .
$$

Then

$$
\begin{aligned}
|x(t)|= & \left|\kappa \mathcal{A}_{1}(x, y)(t)\right| \\
\leq & I^{\alpha_{1}}\left(\left(C_{0}+C_{1}\|x\|+C_{2}\|y\|\right) \frac{1}{p^{*}} I^{\alpha_{2}}(1)\right)(T)+\left(\frac{r^{*}}{p^{*}}\right)\|x\| I^{\alpha_{1}}(1)(T) \\
& +\left(\frac{\gamma_{1}}{\gamma_{1}+1}\left(C_{0}+C_{1}\|x\|+C_{2}\|y\|\right) I^{\alpha_{2}}(1)(T)+\frac{\left|\eta_{1}\right|}{\gamma_{1}+1}(T)\|x\|\right) I^{\alpha_{1}}\left(\frac{1}{p^{*}}\right)(T) \\
& +\frac{1}{2}\left[I^{\alpha_{1}}\left(\left(C_{0}+C_{1}\|x\|+C_{2}\|y\|\right) \frac{1}{p} I^{\alpha_{2}}(1)(T)\right)+\left(\frac{r^{*}}{p^{*}}\|x\|\right) I^{\alpha_{1}}(1)(T)\right. \\
& \left.+\left(\frac{\gamma_{1}}{\gamma_{1}+1}\left(C_{0}+C_{1}\|x\|+C_{2}\|y\|\right) I^{\alpha_{2}}(1)(T)+\frac{\left|\eta_{1}\right|}{\gamma_{1}+1}\|x\|\right)\left|\rho_{1}\right|\right] \\
= & \left(C_{0}+C_{1}\|x\|+C_{2}\|y\|\right) M_{1}+M_{2}\|x\|
\end{aligned}
$$

and

$$
\begin{aligned}
|y(t)|= & \left|\kappa \mathcal{A}_{2}(x, y)(t)\right| \\
\leq & I^{\beta_{1}}\left(\left(D_{0}+D_{1}\|x\|+D_{2}\|y\|\right) \frac{1}{q} I^{\beta_{2}}(1)(T)\right)+\left(\frac{s^{*}}{q^{*}}\right)\|y\| I^{\beta_{1}}(1)(T) \\
& +\left(\frac{\gamma_{2}}{\gamma_{2}+1}\left(D_{0}+D_{1}\|x\|+D_{2}\|y\|\right) I^{\beta_{2}}(1)(T)+\frac{\left|\eta_{2}\right|}{\gamma_{2}+1}(T)\|y\|\right) I^{\beta_{1}}\left(\frac{1}{q^{*}}\right)(T) \\
& +\frac{1}{2}\left[I^{\beta_{1}}\left(\left(D_{0}+D_{1}\|x\|+D_{2}\|y\|\right) \frac{1}{q^{*}} I^{\beta_{2}}(1)(T)\right)+\left(\frac{s^{*}}{q^{*}}\|y\|\right) I^{\beta_{1}}(1)(T)\right. \\
& \left.+\left(\frac{\gamma_{2}}{\gamma_{2}+1}\left(D_{0}+D_{1}\|x\|+D_{2}\|y\|\right) I^{\beta_{2}}(1)(T)+\frac{\left|\eta_{2}\right|}{\gamma_{2}+1}\|y\|\right)\left|\rho_{2}\right|\right] \\
= & \left(D_{0}+D_{1}\|x\|+D_{2}\|y\|\right) M_{3}+M_{4}\|y\| .
\end{aligned}
$$

Hence

$$
\begin{aligned}
& \|x\| \leq\left(C_{0}+C_{1}\|x\|+C_{2}\|y\|\right) M_{1}+M_{2}\|x\|, \\
& \|y\| \leq\left(D_{0}+D_{1}\|x\|+D_{2}\|y\|\right) M_{3}+M_{4}\|y\| .
\end{aligned}
$$

Then we have

$$
\|x\|+\|y\| \leq C_{0} M_{1}+D_{0} M_{3}+\left(C_{1} M_{1}+M_{2}+D_{1} M_{3}\right)\|x\|+\left(C_{2} M_{1}+D_{2} M_{3}+M_{4}\right)\|y\| .
$$


Consequently,

$$
\|(x, y)\| \leq \frac{C_{0} M_{1}+D_{0} M_{3}}{J^{*}},
$$

for any $t \in[0, T]$, where $J^{*}=\min \left\{1-J_{1}, 1-J_{2}\right\}$, which proves that $\mathcal{J}$ is bounded. Hence, by Lemma 3.1, the operator $\mathcal{A}$ has at least one fixed point. So, problem (1.2)-(1.3) has at least one solution on $[0, T]$. The proof is completed.

Let $J_{3}=1-\left(C_{1} M_{1}+D_{1} M_{3}\right), J_{4}=1-\left(C_{2} M_{1}+D_{2} M_{3}\right), J_{5}=1-\left(a_{1} C_{1}+a_{2}+a_{3} D_{1}\right)$ and $J_{6}=1-\left(a_{1} C_{2}+a_{4}+a_{3} D_{2}\right)$. We have the following results.

Corollary 3.3 Suppose that conditions $\left(H_{3}\right)$ and $\left(H_{4}\right)$ of Theorem 3.2 hold. If $J_{3}<1$ and $J_{4}<1$, then system (1.4) with (1.3) has at least one solution on $[0, T]$.

Corollary 3.4 Assume that conditions $\left(H_{3}\right)$ and $\left(H_{4}\right)$ of Theorem 3.2 are fulfilled. If $J_{5}<1$ and $J_{6}<1$, then system (1.5) with (1.3) has at least one solution on $[0, T]$.

\section{Examples}

In this section, we present two examples to illustrate our results.

Example 4.1 Consider the following system of generalized Sturm-Liouville and Langevin fractional differential equations subject to anti-periodic boundary conditions:

$$
\left\{\begin{array}{rlrl}
D^{3 / 5} & \left(\left[\left(t^{3 / 2}+8\right) D^{2 / 3}+\left(t^{2 / 7}-1\right)\right] x(t)\right)=\frac{|x| \sin ^{2}(2 \pi t)}{(4-t)^{2}}\left(\frac{|x|}{|x|+4}+1\right)+\frac{|y|+1}{(4-t)^{2}} \\
& 0<t<2 \\
D^{2 / 5} & \left(\left[\left(t^{5 / 3}+7\right) D^{3 / 4}+\left(t^{3 / 10}-1\right)\right] y(t)\right)=\frac{|x|}{(7+t)^{2}}+\frac{\cos ^{2}(\pi t)}{(5-t)}\left(\frac{|y|}{|y|+5}+1\right), \\
& <t<2, & \\
x(0) & =-x(2), & D^{2 / 3} x(0)=-D^{2 / 3} x(2), \\
y(0) & =-y(2), & D^{3 / 4} y(0)=-D^{3 / 4} y(2) .
\end{array}\right.
$$

Here $\alpha_{1}=2 / 3, \alpha_{2}=3 / 5, \beta_{1}=3 / 4, \beta_{2}=2 / 5, T=2, p(t)=t^{3 / 2}+8, q(t)=t^{5 / 3}+7, r(t)=t^{2 / 7}$ $1, s(t)=t^{3 / 10}-1, f(t, x, y)=\left(\left(|x| \sin ^{2}(2 \pi t)\right) /(4-t)^{2}\right)(|x| /(|x|+4)+1)+\left((|y|+1) /(4-t)^{2}\right)$ and $g(t, x, y)=\left(|x| /(7+t)^{2}\right)+\left(\cos ^{2}(\pi t) /(5-t)\right)((|y| /(|y|+5))+1)$. From the above information, we can find that $p^{*}=8, q^{*}=7, r^{*}=0.21901, s^{*}=0.23114, \gamma_{1}=0.73879, \gamma_{2}=0.6797, \eta_{1}=$ $1.16180, \eta_{2}=1.15901$. Since

$$
\left|f\left(t, x_{1}, y_{1}\right)-f\left(t, x_{2}, y_{2}\right)\right| \leq \frac{1}{64}\left|x_{1}-x_{2}\right|+\frac{1}{16}\left|y_{1}-y_{2}\right|
$$

and

$$
\left|g\left(t, x_{1}, y_{1}\right)-g\left(t, x_{2}, y_{2}\right)\right| \leq \frac{1}{49}\left|x_{1}-x_{2}\right|+\frac{1}{25}\left|y_{1}-y_{2}\right|,
$$

the assumptions of Theorem 3.1 are satisfied with $m_{1}=1 / 64, m_{2}=1 / 16, n_{1}=1 / 49, n_{2}=$ $1 / 25, M_{1}=0.63199, M_{2}=0.29248, M_{3}=0.68084, M_{4}=0.35986$. Thus

$$
L_{1}=\left(m_{1}+m_{2}\right) M_{1}+M_{2}=0.34185, \quad L_{2}=\left(n_{1}+n_{2}\right) M_{3}+M_{4}=0.40098
$$


Therefore, we have $L_{1}+L_{2}=0.74283<1$. Hence, by Theorem 3.1, problem (4.1) has a unique solution on $[0,2]$.

Example 4.2 Consider the following system of generalized Sturm-Liouville and Langevin fractional differential equations subject to anti-periodic boundary conditions:

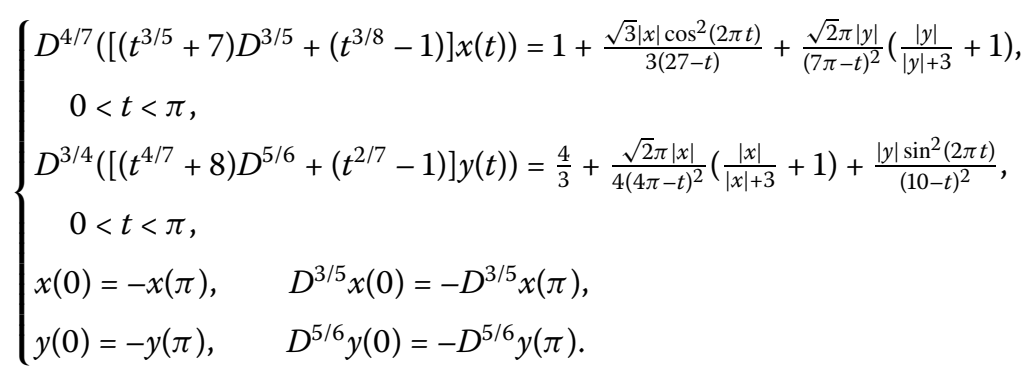

Here $\alpha_{1}=3 / 5, \alpha_{2}=4 / 7, \beta_{1}=5 / 6, \beta_{2}=3 / 4, T=\pi, p(t)=t^{3 / 5}+7, q(t)=t^{4 / 7}+8, r(t)=$ $t^{3 / 8}-1, s(t)=t^{2 / 7}-1, f(t, x, y)=1+\left(\left(\sqrt{3}|x| \cos ^{2}(2 \pi t)\right) /(3(27-t))\right)+((\sqrt{2} \pi|y|) /(7 \pi-$ $\left.t)^{2}\right)((|y| /(|y|+3)+1))$ and $g(t, x, y)=(4 / 3)+\left((\sqrt{2} \pi|x|) /\left(4(4 \pi-t)^{2}\right)\right)((|x| /(|x|+3)+1))+$ $\left(\sin ^{2}(2 \pi t) / 100\right)$. From all the information, we can find that $p^{*}=7, q^{*}=8, r^{*}=0.53614$ and $s^{*}=0.38689$. It is obvious that

$$
\left|f\left(t, x_{1}, x_{2}\right)\right| \leq 1+\frac{\sqrt{3}}{81}\left|x_{1}\right|+\frac{\sqrt{2}}{49 \pi}\left|x_{2}\right|
$$

and

$$
\left|g\left(t, x_{1}, x_{2}\right)\right| \leq \frac{4}{3}+\frac{\sqrt{2}}{64 \pi}\left|x_{1}\right|+\frac{1}{100}\left|x_{2}\right|
$$

Then the assumptions of Theorem 3.2 are satisfied with $C_{0}=1, C_{1}=\sqrt{3} / 81, C_{2}=\sqrt{2} / 49 \pi$, $D_{0}=4 / 3, D_{1}=\sqrt{2} / 64 \pi, D_{2}=1 / 100$, and

$$
\begin{aligned}
& J_{1}=1-\left(C_{1} M_{1}+M_{2}+D_{1} M_{3}\right)=0.32897<1, \\
& J_{2}=1-\left(C_{2} M_{1}+M_{4}+D_{2} M_{3}\right)=0.39883<1 .
\end{aligned}
$$

Therefore, all the conditions of Theorem 3.2 hold true; and consequently, by the conclusion of Theorem 3.2, problem (4.2) has at least one solution on $[0, \pi]$.

\section{Competing interests}

The authors declare that they have no competing interests.

\section{Authors' contributions}

All authors contributed equally in this article. They read and approved the final manuscript.

\section{Author details}

${ }^{1}$ Nonlinear Dynamic Analysis Research Center, Department of Mathematics, Faculty of Applied Science, King Mongkut's University of Technology North Bangkok, Bangkok, 10800, Thailand. ²Department of Mathematics, University of loannina, Ioannina, 451 10, Greece. ${ }^{3}$ Nonlinear Analysis and Applied Mathematics (NAAM)-Research Group, Department of Mathematics, Faculty of Science, King Abdulaziz University, P.O. Box 80203, Jeddah, 21589, Saudi Arabia. ${ }^{4}$ Centre of Excellence in Mathematics, CHE, Sri Ayutthaya Rd., Bangkok, 10400, Thailand. 


\section{Acknowledgements}

This research is partially supported by the Center of Excellence in Mathematics, the Commission on Higher Education, Thailand.

Received: 22 September 2016 Accepted: 10 February 2017 Published online: 27 February 2017

\section{References}

1. Podlubny, I: Fractional Differential Equations. Academic Press, San Diego (1999)

2. Kilbas, AA, Srivastava, HM, Trujillo, JJ: Theory and Applications of Fractional Differential Equations. North-Holland Mathematics Studies, vol. 204. Elsevier, Amsterdam (2006)

3. Agarwal, RP, Zhou, Y, He, Y: Existence of fractional neutral functional differential equations. Comput. Math. Appl. 59, 1095-1100 (2010)

4. Ahmad, B, Ntouyas, SK, Tariboon, J: Existence results for mixed Hadamard and Riemann-Liouville fractional integro-differential equations. Adv. Differ. Equ. 2015, 293 (2015)

5. Ahmad, B, Nieto, JJ: Riemann-Liouville fractional integro-differential equations with fractional nonlocal integral boundary conditions. Bound. Value Probl. 2011, 36 (2011)

6. Ahmad, B, Ntouyas, SK, Alsaedi, A: New existence results for nonlinear fractional differential equations with three-point integral boundary conditions. Adv. Differ. Equ. 2011, Article ID 107384 (2011)

7. Tariboon, J, Ntouyas, SK, Thiramanus, P: Riemann-Liouville fractional differential equations with Hadamard fractional integral conditions. Int. J. Appl. Math. Stat. 54, 119-134 (2016)

8. Ahmad, B, Ntouyas, SK, Alsaedi, A: A study of nonlinear fractional differential equations of arbitrary order with Riemann-Liouville type multistrip boundary conditions. Math. Probl. Eng. 2013, Article ID 320415 (2013)

9. Ahmad, B, Nieto, JJ: Boundary value problems for a class of sequential integrodifferential equations of fractional order. J. Funct. Spaces Appl. 2013, Article ID 149659 (2013)

10. Zhang, L, Ahmad, B, Wang, G, Agarwal, RP: Nonlinear fractional integro-differential equations on unbounded domains in a Banach space. J. Comput. Appl. Math. 249, 51-56 (2013)

11. Liu, X, Jia, M, Ge, W: Multiple solutions of a p-Laplacian model involving a fractional derivative. Adv. Differ. Equ. 2013, $126(2013)$

12. Ntouyas, SK, Obaid, M: A coupled system of fractional differential equations with nonlocal integral boundary conditions. Adv. Differ. Equ. 2012, 130 (2012)

13. Su, X: Boundary value problem for a coupled system of nonlinear fractional differential equations. Appl. Math. Lett. 22, 64-69 (2009)

14. Sun, J, Liu, Y, Liu, G: Existence of solutions for fractional differential systems with antiperiodic boundary conditions. Comput. Math. Appl. 64, 1557-1566 (2012)

15. Tariboon, J, Ntouyas, SK, Thiramanus, P: Impulsive quantum difference systems with boundary conditions. Adv. Differ Equ. 2015, 163 (2015)

16. Senol, B, Yeroglu, C: Frequency boundary of fractional order systems with nonlinear uncertainties. J. Franklin Inst. 350 , 1908-1925 (2013)

17. Henderson, J, Luca, R: Existence and multiplicity of positive solutions for a system of fractional boundary value problems. Bound. Value Probl. 2014, 60 (2014)

18. Ahmad, B, Ntouyas, SK: Existence results for a coupled system of Caputo type sequential fractional differential equations with nonlocal integral boundary conditions. Appl. Math. Comput. 266, 615-622 (2015)

19. Shah, K, Khalil, H, Khan, RA: Investigation of positive solution to a coupled system of impulsive boundary value problems for nonlinear fractional order differential equations. Chaos Solitons Fractals 77, 240-246 (2015)

20. Shah, K, Khan, RA: Existence and uniqueness of positive solutions to a coupled system of nonlinear fractional order differential equations with anti periodic boundary conditions. Differ. Equ. Appl. 7(2), 245-262 (2015)

21. Shah, K, Ali, A, Khan, RA: Degree theory and existence of positive solutions to coupled systems of multi-point boundary value problems. Bound. Value Probl. 2016, 43 (2016)

22. Wang, J, Fečkan, M, Zhou, Y: Presentation of solutions of impulsive fractional Langevin equations and existence results. Eur. Phys. J. Spec. Top. 222, 1857-1874 (2013)

23. Coffey, WT, Kalmykov, YP, Waldron, JT: The Langevin Equation, 2nd edn. World Scientific, Singapore (2004)

24. Lim, SC, Li, M, Teo, LP: Langevin equation with two fractional orders. Phys. Lett. A 372, 6309-6320 (2008)

25. Lim, SC, Teo, LP: The fractional oscillator process with two indices. J. Phys. A, Math. Theor. 42, Article ID 065208 (2009)

26. Uranagase, M, Munakata, T: Generalized Langevin equation revisited: mechanical random force and self-consistent structure. J. Phys. A, Math. Theor. 43, Article ID 455003 (2010)

27. Denisov, SI, Kantz, H, Hänggi, P: Langevin equation with super-heavy-tailed noise. J. Phys. A, Math. Theor. 43, Article ID 285004 (2010)

28. Lozinski, A, Owens, RG, Phillips, TN: The Langevin and Fokker-Planck equations in polymer rheology. Handb. Numer. Anal. 16(C), 211-303 (2011)

29. Lizana, L, Ambjörnsson, T, Taloni, A, Barkai, E, Lomholt, MA: Foundation of fractional Langevin equation: harmonization of a many-body problem. Phys. Rev. E 81, Article ID 051118 (2010)

30. Yukunthorn, W, Ntouyas, SK, Tariboon, J: Nonlinear fractional Caputo-Langevin equation with nonlocal Riemann-Liouville fractional integral conditions. Adv. Differ. Equ. 2014, 315 (2014)

31. Sudsutad, W, Ntouyas, SK, Tariboon, J: Systems of fractional Langevin equation via Riemann-Liouville and Hadamard types and their fractional integral conditions. Adv. Differ. Equ. 2015, 235 (2015)

32. Tariboon, J, Ntouyas, SK, Thaiprayoon, C: Nonlinear Langevin equation of Hadamard-Caputo type fractional derivatives with nonlocal fractional integral conditions. Adv. Math. Phys. 2014, Article ID 372749 (2014)

33. Rivero, M, Trujillo, JJ, Velasco, MP: A fractional approach to the Sturm-Liouville problem. Cent. Eur. J. Phys. doi:10.2478/s11534-013-0216-2

34. Granas, A, Dugundji, J: Fixed Point Theory. Springer, New York (2003) 